\title{
BMJ Open Quality Optimisation of intravenous fluid prescribing: framework for changing practice through education and audits
}

\author{
James Forryan, Vinita Mishra
}

To cite: Forryan J, Mishra V. Optimisation of intravenous fluid prescribing: framework for changing practice through education and audits.BMJ Open Quality 2017;6:e000187. doi:10.1136/ bmjoq-2017-000187

Received 14 August 2017 Revised 24 September 2017 Accepted 3 October 2017

\section{CrossMark}

Department of Clinical Biochemistry, Royal Liverpool and Broadgreen University Hospitals NHS Trust, Liverpool, UK

Correspondence to Dr James Forryan; james.forryan@me.com

\begin{abstract}
Introduction Intravenous fluids are one of the most commonly prescribed drugs in the hospital setting and yet the practice continues to fall short of National Institute for Health and Care Excellence (NICE) guidelines, with significant gaps in staff knowledge exposing patients to heightened morbidity and mortality.

Aim Following the 2013 publishing of updated NICE guidelines on intravenous fluid prescribing, an intravenous fluid team was formed within the Royal Liverpool University Hospital (RLUH). Their role has been (and continues to be) to overhaul the culture of suboptimal intravenous fluid prescribing within the hospital and, ultimately, to improve patient outcomes. A framework to engender this change has been developed and is offered as an example to other Trusts within which improvement of guideline-compliant intravenous fluid prescribing remains stagnant.
\end{abstract}

Method There have been three principal stages of the project to this point which are best demonstrated in a chronological manner. The period of 2010-2014 allowed for assessment of the issue of intravenous fluid prescribing and analysis of its causes through serial audits and a staffwide survey. From 2015, there has been implementation of several measures (educative, managerial, administrative and technological) within the hospital to foster reproducible and positive change with regards to intravenous fluid prescribing. Finally, between 2016 and 2017, three cycles of a rolling audit based on NICE guidelines have been completed to allow measurement of improvement in intravenous fluid prescribing practice. Conclusion Results have demonstrated a significant improvement in the appropriateness of the intravenous maintenance and replacement fluids prescribed in the hospital since the March 2016 audit. Moreover, a 29 -fold increase has been observed in the use of $4 \%$ dextrose $/ 0.18 \%$ sodium chloride as maintenance fluid (gold standard as per NICE guidelines) since the staff-wide survey of 2015. Despite progress however, adherence to NICE guidelines remains below the recommended $100 \%$ and therefore further work remains to be done.

\section{INTRODUCTION}

Since 2010, a comprehensive series of sequential intravenous fluid audits have been carried out within the Royal Liverpool University Hospital (RLUH), part of the Royal Liverpool and Broadgreen University Hospital Trust (RLBUHT), in an attempt to raise awareness within this area and ensure consistent, accurate and appropriate fluid prescribing. The audits took place in 2010 , 2013, 2014, 2016 and 2017 with an additional hospital-wide staff survey related to intravenous fluids completed in 2015. The team is led by a consultant chemical pathologist and consists of several junior doctors, pharmacists and nurses. The work focused on the hospital population indiscriminately so that all adult patients receiving fluids on both surgical and medical wards were audited by the team.

Of all drugs administered in the hospital, intravenous fluids are one of the most common and yet the importance of well-informed prescribing is often overlooked. ${ }^{1}$ Within the 2013 National Institute for Health and Care Excellence (NICE) guidelines pertaining to intravenous fluid prescribing in hospital, it was noted that one in five patients could suffer complications and morbidity because of sub-optimal intravenous fluid therapy. ${ }^{2}$ Many intravenous fluid prescriptions are hastily written up, often due to time constraints or a perceived lack of importance for the practice. This can lead to inappropriate intravenous fluid composition, duration, documentation and, ultimately, increased morbidity for patients. ${ }^{3}$ Therefore, the objective of our project has been (and will continue to be) to create a culture in which intravenous fluids are prescribed with the care, expertise and safety that would be afforded to any other drug.

Assessment of problem and analysis of cause: 2010-2014

In order to investigate the extent to which the hospital was falling short of the national intravenous fluid prescribing standards (updated by NICE in 2013), ${ }^{2}$ three serial audits were completed between 2010 and 2014. In short, the results demonstrated that wards were consistently failing to reach targets within the realm of fluid prescribing, especially in terms 
of documenting the type, rate and volume of fluid to be administered and then outlining a plan for continued fluid therapy and monitoring in the following 24 hours.

The issue was further highlighted by a hospital-wide staff survey carried out in 2015 involving 136 members of staff. Of this number, 60 were doctors of varying grade, 35 nurses, 11 healthcare assistants, 20 medical students, 3 pharmacists, with the rest made up by various other job descriptions. The results mirrored those of the audits, showing that just $58 \%$ of participants felt adequately prepared to prescribe intravenous fluids, with the same percentage reporting acceptable knowledge of the various fluid compositions. Perhaps most significantly, when asked to complete two maintenance fluid prescriptions, only $4.1 \%$ were prescribed appropriately. When trying to identify a cause for these findings, it was poor staff knowledge within the area, infrequent and inadequate reviews of fluid balance and an endemic trivialisation of intravenous fluid prescribing on the part of many healthcare professionals that seemed to form the basis for the poor performance. Furthermore, the deep-seated custom within the healthcare profession is that tasks such as intravenous fluid prescribing fall within the remit of those most junior, often leading to errors born from inexperience and gaps in clinical knowledge. ${ }^{4}$

\section{INTERVENTION: A MULTIFACETED APPROACH TO IMPROVING INTRAVENOUS FLUID PRESCRIBING PRACTICE: 2015 ONWARDS}

In light of the aforementioned shortfallings, it was clear that several measures would need to be implemented in order to engender positive, and perhaps more importantly sustainable, change with regards to intravenous fluid prescribing within the Trust.

\section{Intravenous fluid educational week}

In order to lay the groundwork for the project and establish a baseline level of awareness, the intravenous fluid team arranged an educational week in the hospital in 2015. Magnification of the issue was achieved through having ward-based on-the-spot teaching and intravenous fluid screen-savers available throughout the week. The week concluded with a presentation in Grand Round dedicated to intravenous fluids and given by the intravenous fluid team. The session highlighted the risks conferred by poor intravenous fluid prescribing and described several ways in which staff could take measures to improve their own practice, and that of their colleagues. This included bringing attention to the Trust intravenous fluid policy and where it could be accessed. The presentation concluded by summarising the educational week survey results (as above under 'Assessment of problem and analysis of cause: 2010-2014'). Furthermore, at this stage, a bulk email explaining the key issues in relation to intravenous fluid prescribing in the hospital was sent to all consultants via the Medical Director.

\section{Intravenous fluid flow chart}

All hospital trusts are expected to offer a comprehensive intravenous fluid policy based on NICE guidelines but these are usually very word-heavy, non-user friendly documents, thus often remaining foreign to most hospital staff. Consequently, an 'IV fluid flow chart' (online supplementary appendix 1) was created from the hospital policy on intravenous fluids that portrayed the salient information in a digestible and clear manner. These intravenous fluid flow charts were disseminated throughout the Trust in the form of posters on the wards, digital versions in both the laboratory and junior doctors' electronic emergency handbooks and screen-savers on computers. In tandem with the poster was the implementation of a 24-hour intravenous fluid sticker within the Trust's electronic notes system. This was created as a secondary addition to new protocol following the discovery that several central aspects of fluid prescribing practice were largely ignored in the Trust. These included assessments of patients' weight, serum urea and electrolytes and clinical condition prior to intravenous fluid prescription, and the formulation of prospective 24-hour intravenous fluid plans. With this in mind, the sticker was added to the electronic notes system to act as a prompt for clinicians.

\section{Formal intravenous fluid teaching}

While the above were rolled out on a Trust-wide level, promoting an ubiquitous increase in awareness of the issue, it was very important to implement targeted teaching sessions for the various staff directly (and indirectly) involved in intravenous fluid prescribing. Therefore, the intravenous fluid team began delivering teaching sessions on intravenous fluids to all levels of healthcare staff including undergraduate students, nurses, pharmacists and all grades of junior doctor. These sessions are delivered in many settings, ranging from the hospital induction programme for foundation doctors to specialty trainee teaching. The teaching itself involves a 1-hour session that introduces the participant to the inception of intravenous fluid therapy and the way in which it has evolved over time to be both invaluable and, to some degree, risk-associated. It then moves on to relate the pathophysiology of sodium and water homoeostasis in hospitalised patients so that (at least on a basic level) the healthcare staff acquire an understanding of the implications of intravenous fluids in fluid and electrolyte disturbances in acute illness. Finally, the teaching applies this knowledge by explaining the intrinsic link between the indications for intravenous fluids (eg, maintenance, resuscitation, replacement) and the type of intravenous fluid to be prescribed (eg, Hartmann's, normal saline, saline/dextrose, etc) in the patient. Essentially, the session provides junior doctors and staff with a new-found confidence within the area, ultimately leading to improved intravenous fluid prescribing practice. 


\section{Informal intravenous fluid teaching}

Informal teaching around the topic of intravenous fluids was (and still is) provided on the wards by those staff directly involved in the intravenous fluid audit to further ingrain good practice. Furthermore, sustainability is integral to the intravenous fluid project and one-off teaching sessions will quickly be forgotten without repetition of the topic. Therefore, significant effort was made to provide a cyclical manner of teaching in which following one full round of the hospital staff, the intravenous fluid team began the process afresh (as though painting the Forth bridge), re-educating staff on the same topics so that the educative process was reinforced and the information remained topical.

\section{MEASUREMENT OF IMPROVEMENT: AUDITS 2016-2017}

As alluded to above, three cycles (March and September 2016 and May 2017) of a rolling audit have been completed since implementation of the 2013 NICE guidelines (CG174) on intravenous fluids. The objective of the audits themselves was to acquire cross-sectional data in relation to the quality of fluid prescribing in the RLUH using NICE guidance as a standard (table 1). This in turn allowed for identification of improvements, lingering deficiencies and implementation of necessary changes to practice.

The rolling audit has been carried out by the junior doctors within the intravenous fluid team. As junior doctors are responsible for intravenous fluid prescribing in the hospital, their involvement has the dual advantage of improving their understanding within this area, with the upshot of optimal intravenous fluid therapy in hospital patients. During each cycle, the junior doctors retrospectively collated intravenous fluid data from the previous 24 hours on medical and surgical wards within the hospital including the acute medical unit (AMU) and surgical units. To ensure appropriate and relevant data collection, an audit tool was created using the NICE guidelines on intravenous fluids as a blueprint (online supplementary appendix 2). The two cycles in 2016 were carried out on just 1 day during a week. The May 2017 cycle however took place over several weeks with the medical and surgical wards being audited on 4 separate days and AMU being audited daily for 2 weeks. For this reason, sample size was much larger in the 2017 cycle $(n=162)$ in comparison to the March $(n=29)$ and September $(n=45)$ cycles of 2016.

\section{ANALYSIS OF 2017 AUDIT RESULTS AS PER NICE STANDARDS ON INTRAVENOUS FLUIDS}

Standard 1: The patient's fluid and electrolyte needs are assessed as part of every ward review

We assessed this standard to have been completed if a clear link was documented in the notes between the patient's clinical assessment and the need for intravenous fluids, and treatment with intravenous fluids was included in the plan. This was achieved in $72 \%$ of cases.

Standard 2: The following information is included in the intravenous fluid prescription: the type, rate and volume of fluid to be administered

Results indicated that the type and volume of intravenous fluid was documented in $100 \%$ of cases and the rate in $92 \%$ of cases.

Standard 3: Patients have an intravenous fluid management plan which should include details of the fluid and electrolyte prescription over the next 24 hours, details of assessment (urea and electrolytes, weight, fluid balance) and details of the monitoring plan

The details of prospective 24 hours fluid and electrolyte prescriptions were only completed in $23 \%$ of cases, with many intravenous fluid plans extending no further than the phrase 'IV fluids'. In terms of patient assessment, urea

Table 1 Standards for intravenous fluid choice as per National Institute for Health and Care Excellence (NICE) guidelines

\section{Intravenous fluid indication}

Maintenance $\quad$ 1. $25-30 \mathrm{~mL} / \mathrm{kg} /$ day of water.

2. Approximately $1 \mathrm{mmol} / \mathrm{kg} /$ day of potassium, sodium and chloride.

Approximately $50-100 \mathrm{~g} /$ day of glucose to limit starvation ketosis.(In other words, $0.18 \%$ normal saline with $4 \%$ dextrose with no more than $1 \mathrm{~L}$ of either Hartmann's or normal saline per 24 hours.)

Resuscitation

1. The cause of the fluid deficit is identified.

2. A fluid bolus of $500 \mathrm{~mL}$ of crystalloids is given. (In other words, a bolus of Hartmann's or normal saline.)

3. Patients who have received initial fluid resuscitation are reassessed using the ABCDE approach.

4. Patients who have already been given $>2000 \mathrm{~mL}$ of crystalloids and still need fluid resuscitation after reassessment receive expert help.

5. Patients who have not had $>2000 \mathrm{~mL}$ of crystalloids and who still need fluid resuscitation after reassessment receive $250-500 \mathrm{~mL}$ of crystalloids and have a further reassessment using the ABCDE approach.

Replacement Not listed as standard in NICE guidelines.

Intravenous fluid of choice for losses should be normal saline or Hartmann's. 
COMPARISON OF APPROPRIATE

INTRAVENOUS (IV) FLUIDS PRESCRIBED IN

THREE AUDITS(2016-2017)

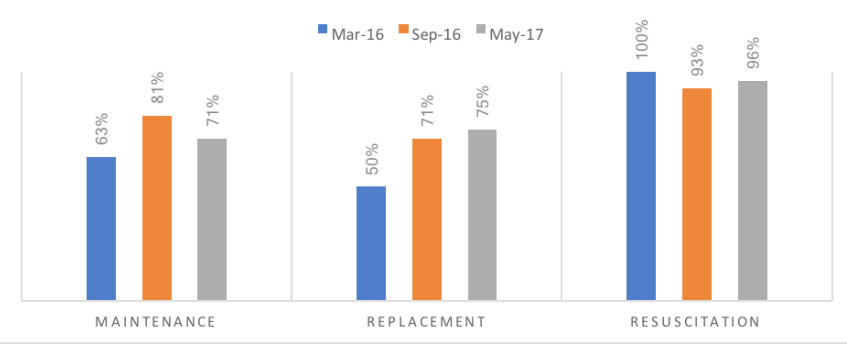

Figure 1 Comparison of percentage compliance with National Institute for Health and Care Excellence intravenous fluid standards (100\% compliance) for maintenance, replacement and resuscitation fluids across audit patients.

and electrolytes were checked in $87 \%$ of cases, weight had been documented in $68 \%$ of cases and fluid balance fully and partially completed in $64 \%$ and $27 \%$ of cases, respectively.

\section{Standard 4: Correct prescription of intravenous maintenance fluids}

Intravenous maintenance fluids were prescribed appropriately in $71 \%$ of patients $(n=103)$ (table 1$)$.

\section{Standard 5: Correct prescription of intravenous resuscitation fluids}

Intravenous resuscitation fluids were prescribed appropriately in $96 \%$ of patients $(n=29)$ (table 1$)$.

\section{Not within NICE standards: correct prescription of intravenous replacement fluids}

Intravenous replacement fluids were prescribed appropriately in $75 \%$ of cases $(n=63)$ (table 1$)$.

\section{4\% DEXTROSE/0.18\% SODIUM CHLORIDE AS MAINTENANCE FLUID}

It is also important to add that since the 2015 intravenous fluid education week and staff survey, the rate at which the gold standard maintenance fluid (as per NICE guidelines) $4 \%$ dextrose $/ 0.18 \%$ sodium chloride is prescribed within the hospital has increased exponentially. In numerical terms, an almost 29-fold increase in the correct use of $4 \%$ dextrose $/ 0.18 \%$ sodium chloride has been observed.

\section{CONCLUSION}

Several positive changes to IV fluid practice in the hospital have arisen because of the work of the IV fluid team. One of these is a marked improvement in the appropriateness of

Table 2 Use of $4 \%$ dextrose- $0.18 \%$ saline bags as maintenance fluid in Royal Liverpool University Hospital

\begin{tabular}{llll}
\hline Year & $\mathbf{2 0 1 5}$ & $\mathbf{2 0 1 6}$ & $\mathbf{2 0 1 7}$ \\
\hline Grand total of bags & 680 & 8930 & 19540 \\
\hline
\end{tabular}

\section{APPROPRIATENESS OF INTRAVENOUS (IV) \\ FLUIDS PRESCRIBED ON WEEKDAYS VS WEEKENDS}

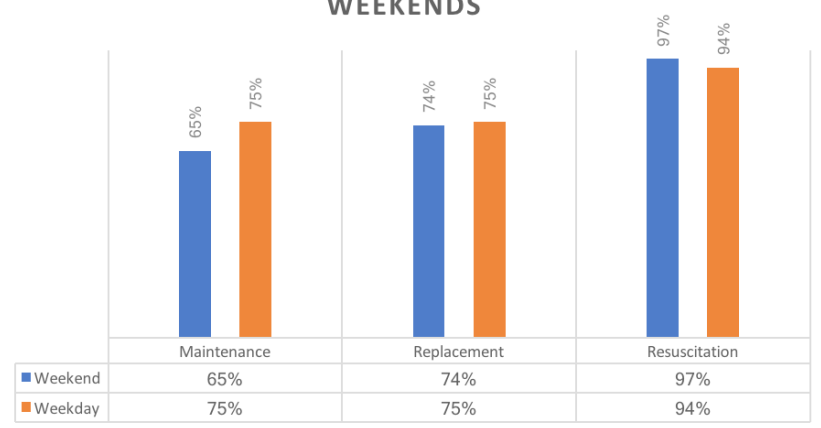

Figure 2 Comparison of percentage compliance with National Institute for Health and Care Excellence intravenous fluid standards (100\% compliance) for maintenance, replacement and resuscitation fluids on weekdays versus weekends.

the IV maintenance and replacement fluids prescribed since the March 2016 audit (figure 1). Moreover, a sharp increase in the use of $4 \%$ dextrose $/ 0.18 \%$ sodium chloride within the Trust has been observed (table 2) which suggests a greater awareness of guideline-orientated IV fluid prescribing. It should also be noted that there was almost no discrepancy between the data collected on weekdays compared with weekends (figure 2). It is junior doctors to whom the task of IV fluid prescribing falls and therefore the results would indicate that there exists a better understanding of the relationship between clinical indication and IV fluid choice within this cohort. Moving forwards the hope would be that a ripple effect is seen, whereby these junior doctors continue to prescribe IV fluids in a correct and safe manner, whilst educating those around them.

Limitations are potentially highlighted when our study is compared with a similar project carried out in $2014^{5}$ (after inception of our project in 2010). The team based in Auckland carried out a double-blinded randomised controlled trial measuring the improvement in intravenous fluid practice following introduction of educational intervention in the study group compared with a control group. Introduction of a control group is a viable consideration for our project moving forwards as it would allow for statistical analysis to assess significance and would add further weight to our results.

Regardless of the progress made so far, there remain significant obstacles, principally the maintaining of interest in the project and its sustainability, that will need to be navigated. However, while adherence to NICE guidelines

\section{Key messages}

There has been an improvement in prescribing appropriate intravenous fluids since the March 2016 audit and consistency since the September 2016 audit.

- The audit reflects that the results on weekends are comparable to those of weekdays.

- 29-fold increase in use of dextrose/saline within the Trust since intravenous fluid education began in 2015 . 
remains below 100\% (NICE recommends $100 \%$ compliance), there will continue to be scope for further progress. Nevertheless, the plan for biannual audits as part of the rolling project, cyclical lectures on intravenous fluids given to staff throughout the year and on-the-spot ward teaching will all work synergistically to help overcome the challenges moving forwards.

\section{Acknowledgements The authors thank for helping in data collection.}

Contributors JF: author and foundation doctor, Helen Campbell, Nageshwaran Vaitehi, Alex Trevaskis, Daniel Gibney, Sid Srinivas, Jenika Patel, Jennifer Chalmers, Louise Zammit, Ashley Holt, Sarah Beresford, Simon West, Rosie Conroy. David Corness member of RLUH audit team, VM: consultant chemical pathologist and director. All listed names were involved in data collection. DC was responsible for collation and initial analysis of data. $\mathrm{HC}$ and VM supervised study design and interpretation. VM oversaw and was involved directly in data collection from the audits, progress to date on the recommendations from those audits, data analysis and data interpretation. VM is also responsible for revisions of the manuscript. JF also carried out data analysis, interpretation and wrote the manuscript, responding to revisions from VM.

Competing interests None declared.

Provenance and peer review Not commissioned; externally peer reviewed.
Open Access This is an Open Access article distributed in accordance with the Creative Commons Attribution Non Commercial (CC BY-NC 4.0) license, which permits others to distribute, remix, adapt, build upon this work non-commercially, and license their derivative works on different terms, provided the original work is properly cited and the use is non-commercial. See: http://creativecommons.org/ licenses/by-nc/4.0/

(c) Published by the BMJ Publishing Group Limited. For permission to use (where not already granted under a licence) please go to http://www.bmj.com/company/ products-services/rights-and-licensing/

\section{REFERENCES}

1. Padhi S, Bullock I, Li L, et al. Intravenous fluid therapy for adults in hospital: summary of NICE guidance. BMJ 2013;347:f7073.

2. National Institute of Health and Care Excellence (NICE). Intravenous Fluid Therapy in Adults in Hospital. 2013; www.nice.org.uk/CG174

3. Lim CT, Dunlop M, Lim CS. Intravenous fluid prescribing practices by foundation year one doctors - a questionnaire study. JRSM Short Rep 2012;3:1-7.

4. Sansom LT, Duggleby L. Intravenous fluid prescribing: Improving prescribing practices and documentation in line with NICE CG174 guidance. BMJ Qual Improv Rep 2014;3:u205899.w2409.

5. Gnanasampanthan V, Porten L, Bissett I. Improving surgical intravenous fluid management: a controlled educational study. ANZ J Surg 2014;84:932-6. 